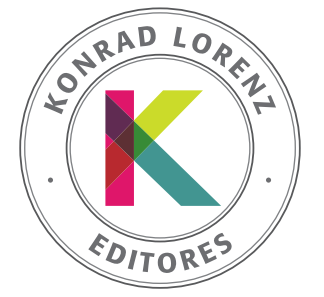

SUMA PSICOLÓGICA

http://editorial.konradlorenz.edu.co/suma-psicologica.html

\title{
INECO Frontal Screening (IFS): una herramienta psicométrica para evaluar la disfunción ejecutiva en policonsumidores
}

\author{
Maryoris Zapata Zabala ${ }^{a}$, Lucila Cárdenas Niño y Jorge Mauricio Cuartas Arias ${ }^{\text {ab* }}$
}

\author{
${ }^{a}$ Universidad Buenaventura, Medellín, Colombia \\ ${ }^{b}$ Departamento de Psicologia, Escuela de Humanidades, Universidad EAFIT, Medellin, Colombia
}

Recibido el 5 de septiembre de 2019; aceptado el 6 de noviembre de 2019

\author{
PALABRAS CLAVE \\ Policonsumidores, \\ neuropsicología, \\ disfunción ejecutiva, \\ INECO frontal screening
}

\section{KEYWORDS}

Polyconsumers, neuropsychology, executive dysfunction, INECO frontal screening
Resumen La presente investigación tiene como objetivo analizar las propiedades psicométricas del INECO frontal screening (IFS) en una población de policonsumidores de la ciudad de Medellín, Colombia. El instrumento se administró a una muestra de 69 sujetos con consumo de sustancias psicoactivas y 69 sujetos control. Los análisis psicométricos evidencian una consistencia aceptable del test: 0.7 ; con respecto a su utilidad diagnóstica se estimó un punto de corte de 22.5 con una sensibilidad del $80 \%$ y una especificidad del $91 \%$. Se encontró evidencia de validez de criterio al comparar el desempeño cognitivo entre el grupo caso y control obteniendo diferencias significativas: $p<0.05$ en cada escala y puntaje total. Por último, se realizó la validez de constructo mediante el análisis factorial exploratorio, encontrando una varianza explicada total de la prueba del $56 \%$, proponiendo tres factores, lo cual contribuye a la eficacia del IFS para identificar déficits cognitivos en población con algún compromiso neurológico o alteraciones neuropsicológicas.

(c) 2019 Fundación Universitaria Konrad Lorenz. Este es un artículo Open Access bajo la licencia CC BY-NC-ND (http://creativecommons.org/licenses/bync-nd/4.0/).

INECO frontal screening (IFS): A test battery to assess executive dysfunction in polyconsumers

Abstract This study analyzed the psychometric properties of the INECO Frontal Screening, IFS, in a population of polyconsumers of the city of Medellín, Colombia. The Psychometric analysis showed an acceptable consistency of the test: 0.7 ; with respect to its diagnostic utility, a cutoff point of 22.5 was estimated with a sensitivity of $80 \%$ and a specificity of $91 \%$. As to its validity, evidence of criterion validity was found when comparing cognitive performance between the case and control group, obtaining significant differences: $p<0.05$, in each scale and total score. Finally, construct validity was performed through exploratory factor analysis, and the total variance explained of the $56 \%$ test was found, suggesting three factors, which contribute to the effectiveness of the IFS test to identify cognitive deficits in people with some type of neurological and neuropsychological dysfunction.

(c) 2019 Fundación Universitaria Konrad Lorenz. This is an open access article under the CC BY-NCND license (http://creativecommons.org/licenses/bync-nd/4.0/).

\footnotetext{
* Autor para correspondencia.

Correo electrónico: mauricio.cuartas@usbmed.edu.co
} 
Según la Oficina de las Naciones Unidas contra el Control de la Droga y el Delito (UNODC, por sus siglas en inglés), cerca de 29 millones de personas que consumen drogas sufren de trastornos mentales (UNODC, 2017). Ello no solo impacta de manera negativa el desarrollo sostenible de los países, sino también el deterioro cognitivo asociado a personas con dependencia al uso de sustancias psicoactivas (SPA), remodela de forma negativa el pronóstico, la evolución de síntomas y la adherencia a procesos terapéuticos exitosos en pro de recuperar estilos de vida saludables.

Para Colombia, el panorama es congruente con el aumento mundial del consumo y el uso problemático de las drogas. Al respecto, los Ministerios de Salud y Justicia y el Observatorio de Drogas de Colombia (ODC), en su última encuesta nacional sobre consumo de drogas, evidencian un incremento en el porcentaje de $8.6 \%$ en el 2008 a $12.17 \%$ en el 2013 en personas que por lo menos recurrieron una vez en la vida al empleo de sustancias ilícitas como marihuana, bazuco, éxtasis o heroína. De igual manera, en el primer estudio poblacional de salud mental en Medellín, entre el 2011 y 2012, los resultados arrojados sobre el consumo de sustancias psicoactivas muestran que el uso en el último año de alguna sustancia ilícita fue reportado por $2.7 \%$ de los encuestados, lo que equivale a cerca de 540.000 personas; siendo el consumo más alto entre los hombres (4.5\%) que entre las mujeres (1.2\%). Entre los grupos de edad con mayor prevalencia de uso de sustancias ilícitas en el último año con 3.4\% está el de 12 a 17 años; al igual que en la mayoría de los países del mundo, la marihuana es la sustancia ilícita de mayor consumo. El $8.0 \%$ de las personas encuestadas informaron haberla consumido al menos una vez en la vida, aproximadamente $13.0 \%$ entre los hombres y $4.0 \%$ entre las mujeres.

Basados en los estudios mencionados, el aumento en el abuso de drogas es un problema especialmente complejo, ya que está modulado por factores de muy diversa índole y se relaciona con la presencia de problemas médicos, familiares, sociales, laborales, económicos, legales y psicológicos asociados a su uso. En los últimos años, se viene desarrollando un sólido marco teórico y empírico sobre las conductas adictivas y su tratamiento, se han producido y estandarizado instrumentos de evaluación cada vez más sensibles, específicos, e intervenciones cada vez más eficaces (Secades \& Fernández, 2003).

Desde esta perspectiva, la neuropsicología ha descrito déficits cognitivos derivados del consumo de drogas en personas con adicción a distintas sustancias y cómo esto afecta la prognosis y evolución de síntomas en procesos de intervención terapéutica (Perry \& Lawrence, 2017); se encuentran prevalencias estimadas entre el 30\%-80\% de deterioro cognitivo para sujetos adictos. No obstante, el desempeño cognitivo estimado desde dominios cognitivos específicos es aún controversial y poco explorado; esto conlleva la necesidad de implementar estrategias de evaluación clínica eficientes en sensibilidad, especificidad y uso del tiempo de evaluación que permitan conocer los grados de afectación de la persona consumidora de sustancias psicoactivas. Hasta ahora, los protocolos de evaluación utilizados en la medición y evaluación de procesos cognitivos en personas policonsumidoras se han enfocado en tareas asociadas al funcionamiento frontal; si bien, estas baterías de evaluación cuentan con buen respaldo teórico y validez convergente en neuropsicología, su aplicación resulta ser compleja y extensa, esta condición limita su uso en la evaluación de muestras clínicas más amplias y, por tanto, en la replicabilidad de los estudios. Además, la gran disparidad de protocolos clínicos no ha contribuido a la generalización de resultados, afectando la validez ecológica, la sensibilidad y especificidad de los test implementados de acuerdo con la población de referencia.

Frente a los inconvenientes que plantea el uso de baterías neuropsicológicas extensas para evaluar la función frontal, el IFS surge como prueba de rastreo cognitivo que podría facilitar la medición y el análisis del rendimiento cognitivo en población policonsumidora; es un test de fácil aplicación que, además, disminuye significativamente el tiempo de evaluación del funcionamiento frontal. La prueba fue diseñada inicialmente por el Instituto de Neurología Cognitiva (INECO), con el propósito de identificar posibles métodos para medir la disfunción cognitiva en pacientes con quejas en su rendimiento cognitivo y su desempeño funcional en tareas que requieren funcionamiento ejecutivo. El instrumento incorpora diferentes pruebas para valorar la función frontal (Báez et al., 2014; Torralva, Roca, Gleichgerrcht, López \& Manes, 2009).

Por tanto, ante la falta de instrumentos válidos, versátiles en el uso del tiempo y confiables para determinar el funcionamiento ejecutivo en sujetos con uso problemático o dependencia de sustancias psicoactivas, evaluamos las propiedades psicométricas del IFS en función a habilitar nuevas herramientas clínicas que contribuyan a mejorar el diagnóstico y la rehabilitación en este tipo de patologías.

\section{Método}

Mediante un muestreo no probabilístico, se seleccionaron 69 casos del programa de rehabilitación de adicciones en la Institución Brújula SM (http://www.brujulasm.com), localizada en la ciudad de Envigado (Antioquia), con antecedentes de consumo de sustancias psicoactivas. Como criterio de referencia para el diagnóstico de policonsumo, se tuvo en cuenta la historia clínica, además de la evaluación clínica realizada por un psiquiatra, psicólogo y neuropsicólogo que participan en el programa de adicciones. Se determinaron aspectos relevantes a la conducta de consumo: tipo de sustancia, frecuencia y el tiempo de consumo. Para el grupo control se incluyeron 89 sujetos, de los cuales, después del proceso de evaluación neurológica, psiquiátrica o fracaso escolar, fueron excluidos 20 (22.47\%) participantes, por no cumplir los criterios de inclusión: edad mayor o igual a 16 años y ausencia de un trastorno psiquiátrico o neurológico; en los criterios de exclusión se tuvo en cuenta que los participantes presentaran dificultades auditivas o visuales e historia de fracaso escolar, quedando 69 participantes $(77.5 \%)$. Todos los participantes tenían una edad mínima de 18 años y accedieron voluntariamente a formar parte de la investigación firmando el consentimiento informado antes de la aplicación del instrumento.

\section{Instrumento}

INECO frontal screening (IFS)

Es un instrumento de rastreo para medir funciones frontales que cumple con los criterios de validez y confiabilidad (Torralva, Roca, Gleichgerrcht, López \& Manes, 2009). Este instrumento consta tres grupos de tareas cognitivas: 
Inhibición de respuestas y cambio de sets: evalúa la habilidad de cambiar de un set cognitivo a otro y la capacidad de inhibir respuestas inapropiadas de manera verbal y motora.

Memoria de trabajo: referida a un sistema cerebral que provee un almacenamiento temporario y manipulación de la información necesaria para otras tareas cognitivas complejas.

El IFS está compuesto por ocho subtest tomados de otros test de función frontal:

- Series motoras (Dubois, Slachevsky, Litvan \& Pillon, 2000): en esta subprueba se le pide al paciente que realice la serie de Luria: "Puño, Canto, Palma”, inicialmente copiando al administrador, y luego repitiendo la serie por sí mismo seis veces.

- Instrucciones conflictivas (Dubois et al., 2000): en esta subprueba se les pide a los sujetos que golpeen la mesa una vez cuando el administrador la golpea dos, y que la golpeen dos veces cuando el administrador la golpea una vez.

- Go-No Go (Dubois et al., 2000): se les indica a los sujetos que cuando el administrador golpee la mesa una vez, ellos deberán golpearla una vez, y cuando el administrador la golpee dos veces, ellos no deberán golpearla.

- Dígitos en orden inverso (Hodges, 1994): en este subtest, el examinador lee una serie de números, en cada ítem se le pide al sujeto que repita la serie en orden inverso. Lea los dígitos de a uno por segundo, bajando levemente la voz en el último dígito de la serie.

- Memoria de trabajo verbal (Hodges, 1994): se le pide al sujeto que enumere los meses del año en orden inverso, empezando por diciembre.

- Memoria de trabajo espacial (Weschler, 1987): en esta prueba, el examinador le presenta al sujeto cuatro cuadrados, los cuales señala siguiendo una secuencia determinada. Se le pide al sujeto que repita la secuencia en orden inverso.

- Capacidad de abstracción (interpretación de refranes) (Hodges, 1994): se eligió este subtest porque en pacientes con lesiones frontales es usual observar dificultades para alejarse de contenidos literales o concretos de una oración y encontrar significado abstracto en ella. En esta se leen los tres refranes y se le pide al sujeto que explique el significado de cada uno de ellos.

- Control inhibitorio verbal (Burgess \& Shallice, 1997): esta tarea inspirada en la del test de Hayling, mide la capacidad de un sujeto de inhibir una respuesta esperada. Esta consiste en seis oraciones donde a cada una de ellas les falta la palabra final; la construcción de ellas está diseñada para que la opción correcta que la completa este sugerida por su contexto. En la primera parte, se leen las tres oraciones donde el sujeto debe completarla lo más rápido posible con la palabra correcta que la completa; en la segunda parte se leen las tres oraciones siguientes donde el sujeto debe completarlas con una palabra diferente al contexto de la oración.

\section{Procedimiento}

En función de analizar las propiedades psicométricas del IFS, se realizaron varias fases:

Fase 1: selección de los participantes por medio de la revisión de historias clínicas para identificar si cumplían o no con los criterios para formar parte de la investigación.

Fase 2: explicación a los participantes de los objetivos de la investigación y firma del consentimiento informado para formar parte de la misma.

Fase 3: aplicación del instrumento IFS, de forma individual a cada uno de los participantes de la investigación, con una duración promedio de 10 minutos en la aplicación del mismo.

Fase 4: recolección de los datos arrojados por la prueba y análisis psicométricos a los mismos por medio de SPSS versión 24.

\section{Resultados}

Uno de los primeros pasos en la validación de la prueba fue la adaptación del instrumento siguiendo los parámetros de la APA (Muñiz, Elosua \& Hambleton, 2013). Para establecer la validez de contenido se tuvieron en cuenta cinco expertos en el constructo psicológico, quienes a través de un formato de calificación de 1 a 3 validaron indicadores como la tarea, instrucción y material de la prueba. El coeficiente utilizado para establecer el nivel de acuerdo, fue el estadístico de Kendall (tabla 1), obteniendo un equivalente a 0.966 con un nivel de significancia de 0.000 , que indica concordancia alta y significativa entre los rangos asignados por los jueces, teniendo en cuenta la categoría equivalencia del constructo, lingüística y cultural y del formato con relación a los indicadores como la tarea, instrucción y material de la prueba. Los expertos no realizaron ningún ajuste al test.

Tabla 1 Evidencia de validez (W de Kendall)

\begin{tabular}{cc}
\hline N & $\mathbf{8}$ \\
\hline W de Kendall $^{\mathrm{a}}$ & 0.966 \\
Chi-cuadrado & 340.177 \\
gl & 44 \\
Sig. asintót. & 0.000 \\
\hline
\end{tabular}

$\mathrm{a}=$ coeficiente de concordancia de Kendall.

Además, se observa que la variable edad no presenta diferencias significativas entre los grupos casos y controles, mientras que las variables sexo y escolaridad sí presentan diferencias estadísticamente significativas (tabla 2)

También se observaron diferencias significativas en la prueba total y cada una de las subpruebas teniendo en cuenta el grupo control y casos (tabla 3 ).

Al analizar cómo se agrupaban los ítems en tres factores, cada uno de los ítems contribuyó con una carga factorial superior a 4, alcanzando una varianza explicada del $56.457 \%$ (tabla 4); asimismo, se pudo determinar una consistencia por cada factor aceptable teniendo en cuenta que son pocos ítems (tabla 5).

\section{Sensibilidad y especificidad del INECO frontal screening}

El análisis de sensibilidad y especificidad mostró una alta capacidad diagnóstica de la prueba con un índice de discriminación bajo la curva de $0.93(p<0.00)$. En la figura 1 se observa la curva de ROC. Este parámetro permite 
contrastar el mejor balance entre sensibilidad y especificidad. Para nuestro estudio se observó un punto de corte correspondiente a 22.5 equivalente al $80 \%$ de sensibilidad y 91\% de especificidad.

Tabla 2 Descripción de las características sociodemográficas de los grupos de análisis

\begin{tabular}{lccc}
\hline & \multicolumn{1}{c}{ Controles } & Caso & Estadístico de prueba \\
\cline { 2 - 4 } & $\mathrm{N}(\%)$ & $\mathrm{N}(\%)$ & $\mathrm{N}=69(50 \%)$ \\
\cline { 2 - 4 } & $\mathrm{N}=69(50 \%)$ & $36.97 \pm 11.49$ & $0.83^{\mathrm{a}}$ \\
\hline Edad $(\mathrm{M} \pm \mathrm{DE})$ & $38.68 \pm 12.62$ & & $\chi^{\mathrm{b}}$ \\
\hline Sexo $\mathrm{N}(\%)$ & & $4(16.7 \%)$ & $12.91^{* * *}$ \\
\hline Hujer & $20(83.3 \%)$ & $65(57 \%)$ & \\
\hline Escolaridad N(\%) & $49(43 \%)$ & & $49.09^{* * *}$ \\
\hline 0 a 5 & & $8(78.3 \%)$ & $47(78.3 \%)$ \\
6 a 11 & $1(11.1 \%)$ & $14(20.3 \%)$ & \\
Mayores de 11 & $13(21.7 \%)$ & $55(79.7 \%)$ & \\
\hline
\end{tabular}

${ }^{\mathrm{a}}=$ prueba T de Student; ${ }^{\mathrm{b}}=$ Chi-cuadrado de Pearson; ${ }^{*+*} \mathrm{p}<0.01$.

Tabla 3 Evidencias de la validez de criterio teniendo en cuenta el puntaje total y subpruebas en cada uno de los grupos

\begin{tabular}{|c|c|c|c|c|c|}
\hline & \multicolumn{2}{|c|}{ Controles } & \multicolumn{2}{|c|}{ Caso } & \multirow{4}{*}{ U de Mann Witney } \\
\hline & & $(\%)$ & & (\%) & \\
\hline & \multicolumn{2}{|c|}{$\mathrm{N}=69(50 \%)$} & \multicolumn{2}{|c|}{$N=69(50 \%)$} & \\
\hline & Media & Desviación & Media & Desviación & \\
\hline Series motoras & 3 & 0 & 3 & 0 & $1962.0^{\text {n** }}$ \\
\hline Instrucciones conflictivas & 3 & 0 & 3 & 0 & $1892.5^{\star \star \star \star}$ \\
\hline Go-No Go & 3 & 0 & 3 & 1 & $1888.0^{\star \star \star}$ \\
\hline Dígitos atrás & 3 & 0 & 3 & 1 & $1394.0^{n * \star}$ \\
\hline Memoria de trabajo verbal & 2 & 0 & 1 & 2 & $1212.0^{* \star *}$ \\
\hline Memoria de trabajo espacial & 3 & 2 & 2 & 1 & $1034.0^{\text {**** }}$ \\
\hline Refranes & 3 & 0 & 2 & 1 & $1192.0^{n \star * *}$ \\
\hline Hayling test & 6 & 1 & 5 & 2 & $1278.5^{\star \star * *}$ \\
\hline Puntaje total & 25 & 2 & 20 & 4 & $304.0^{* \star *}$ \\
\hline
\end{tabular}

$" p<0.00$.

Tabla 4 Evidencias de la validez de constructo

\begin{tabular}{llccc}
\hline Factor & \multicolumn{1}{c}{ Subpruebas } & Carga factorial & Varianza explicada & Varianza total \\
\hline \multirow{2}{*}{ Factor 1} & Series motoras & .772 & 30.447 \\
& Go-No Go & .698 & \\
Factor 2 & Instrucciones conflictivas & .461 & 13.346 \\
& Memoria espacial & .724 & \\
& Memoria verbal & .723 & \\
Factor 3 & Hayling & .547 & 12.664 \\
\end{tabular}


Tabla 5 Confiabilidad de cada subprueba y total

\begin{tabular}{|c|c|c|c|}
\hline Factor & Subpruebas & Confiabilidad & Confiabilidad total \\
\hline \multirow{3}{*}{ Factor 1} & Series motora & \multirow{3}{*}{.500} & \multirow{8}{*}{0.637} \\
\hline & Go-No Go & & \\
\hline & Instrucciones conflictivas & & \\
\hline \multirow{3}{*}{ Factor 2} & Memoria de trabajo espacial & \multirow{3}{*}{.530} & \\
\hline & Dígitos en orden inverso & & \\
\hline & Memoria de trabajo verbal & & \\
\hline \multirow{2}{*}{ Factor 3} & Control inhibitorio verbal & \multirow{2}{*}{.400} & \\
\hline & Capacidad de abstracción & & \\
\hline
\end{tabular}

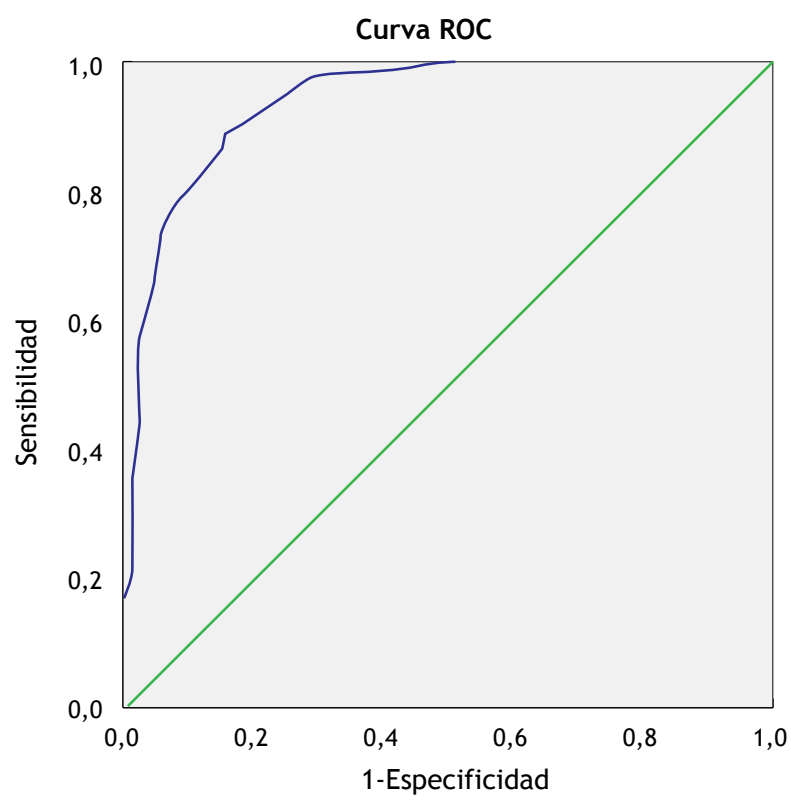

Los segmentos de diagonal se genera mediante empates

Figura 1 Capacidad diagnóstica de la prueba INECO

\section{Discusión}

El uso de protocolos clínicos y test de tamizaje para la evaluación clínica de la disfunción ejecutiva, continúa siendo un propósito relevante en la investigación de los distintos síndromes y trastornos con compromiso neurológico. No obstante, aún son pocos y los problemas psicométricos de las baterías neuropsicológicas utilizadas, conllevan con frecuencia falta de sensibilidad y especificidad; un ejemplo de ello es la evaluación de la fluencia verbal que puede exhibir variaciones con relación a variables sociodemográficas como la edad y la educación formal (Buriel, Gramunt, Bohm, Rodes y Peña-Casanova, 2004). Las ventajas del IFS respeta la medición de las funciones ejecutivas como dominios que interactúan pero que exhiben independencia; además, el uso reducido del tiempo, la fácil administración y puntuación, y su uso en poblaciones latinas sugieren al IFS como un test de tamizaje pertinente, cuyo modelo teórico está bien estimado (Karr et al., 2018; Miyake et al., 2000).
La descripción de las variables sociodemográficas en ambos grupos evidencia una diferencia estadísticamente significativa en cuanto al sexo y la escolaridad (tabla 1). Observándose que en el grupo de casos hay una mayor tendencia de hombres con respecto a las mujeres, lo anterior ha sido expuesto en las conclusiones obtenidas del estudio de López y Rodríguez (López-Larrosa \& Rodríguez-Arias, 2010, 2012), quienes proponen factores de riesgo y de protección y donde se ha observado que los hombres tienen significativamente mayores factores de riesgo tanto personales como contextuales y menos factores protectores respecto al grupo de mujeres; ya que en esta última población puede pensarse que opera el mecanismo de riesgo-protección, incidiendo para ambos casos factores de orden personal y familiar, lo que podría mostrar una mayor dificultad en el grupo masculino donde la protección solo es en el ámbito comunitario. No obstante, diferentes estudios han confirmado las diferencias significativos para género y consumo de drogas ilegales. Además, las diferencias estadísticamente significativas para consumo en ambos sexos están asociadas con el tiempo de inicio, la frecuencia y el estatus de policonsumo. Al respecto, estos resultados concuerdan con lo observado por diferentes investigaciones que han sugerido que la frecuencia de consumo y el policonsumo son diferentes en hombres y mujeres (Fernández-Montalvo, López-Goni, Azanza, Arteaga \& Cacho, 2017; Hser, Huang, Teruya \& Anglin, 2003). Este hallazgo tiene implicaciones clínicas relacionadas con la severidad, la abstinencia posconsumo y la reincidencia en las drogas, entre otras variables sociodemográficas de interés para estudios posteriores que permitan comparar los resultados terapéuticos y los factores predictores asociados en ambos sexos desde su rendimiento cognitivo.

Con respecto a la escolaridad, hay una mayor proporción de consumo en sujetos entre los 6 y 11 años de escolaridad. Lo anterior puede asociarse con la edad de inicio del consumo de SPA, el cual es frecuente entre los 10 y los 13 años de edad, factor que puede inducir a la desescolarización. Otros factores han sido asociados al consumo temprano y deserción escolar, tales como la disfunción familiar, el consumo por parte de alguna de las figuras parentales y los contextos donde los chicos reciben influencias directas del grupo de pares. Asimismo, este incremento en el consumo asociado con los años de escolaridad también está relacionado con la variable sexo. Algunos estudios han 
determinado que hay un mayor incremento en los hombres con $37 \%$ respecto de las mujeres con $34 \%$; señalando al alcohol con $34 \%$ como la sustancia legal más consumida y las sustancias ilegales con $3 \%$ de consumo (Acosta-Barreto, Juárez-Acosta \& Cuartas-Arias, 2018)(Espinosa S. K., 2016 \#3164; Acosta Barreto, 2018 \#8).

Cabe resaltar que la validez entendida como una evidencia para la interpretación de las puntuaciones (Fisher, 2011), en el análisis de contenido se observa acuerdo entre los jueces para mantener las instrucciones, tareas y material, como se plantean en la prueba original. Esto indica, a juicio de expertos, que aspectos relacionados con el contenido del dominio, claridad de la instrucción y relevancia de las tareas para los objetivos de la estimación, son suficientes, importantes y coherentes, lo cual garantiza la pertinencia de las inferencias (Muñiz, 2000) (tablas 2 y 3). Con respecto a la valoración de las diferencias en los diferentes niveles del constructo entre los grupos (caso - control), se observa una clara capacidad discriminativa de cada una de las subpruebas para diferenciar el desempeño entre los dos grupos, lo cual confirma la evidencia de validez referida a criterio externo (Prieto, Delgado, Perea \& Ladera, 2010).

Se describieron resultados similares en el estudio de análisis de las propiedades psicométricas del IFS en pacientes con demencia, en población chilena (Ihnen, Antivilo, Muñoz-Neira \& Slachevskya, 2013), donde la validación de expertos no sugiere cambios o ajustes de contenido con respecto a la instrucción, tarea o material.

Además, para la valoración de las diferencias en cada uno de los niveles del constructo entre los grupos (caso control), se observó una clara capacidad discriminativa de cada una de las subpruebas para diferenciar el desempeño entre los dos grupos (tabla 3), lo cual confirma la evidencia de validez referida a criterio externo y en congruencia con lo formulado por el grupo de Prieto et al. (2010). En otros estudios, cada uno de los dominios ha sido asociado como constructo relevante para diferenciar el funcionamiento ejecutivo y sugestivo y para revelar la disfunción neuropsicológica para la dependencia de sustancias psicoactivas. En particular, el rendimiento en la tarea de series motoras y la abstracción están involucradas con el funcionamiento de las áreas medial, dorsolateral y posterior de la corteza prefrontal (Cunha, Nicastri, De Andrade \& Bolla, 2010; Dubois et al., 2000). En esta misma vía de hallazgos, las tareas relacionadas con memoria espacial, se conectan con el desempeño en la corteza prefrontal medial a través de los receptores dopaminérgicos; esto contrasta con el argumento asociado con el policonsumo y la presencia de un deterioro cognitivo relacionado con esta zona cortical (De Melo-Rodrigues, Conti \& Nakamura-Palacios, 2011; Wesley, Lile, Fillmore \& Porrino, 2017). De manera similar, el bajo desempeño en tareas de control inhibitorio ha sido frecuentemente asociado al uso y dependencia de sustancias (Noël, Brevers \& Bechara, 2013; Smith, Jones, Bullmore, Robbins \& Ersche, 2013; Yücel, Lubman, Solowij \& Brewer, 2007). Generalmente en jóvenes en riesgo de abuso y dependencia de sustancias, se infiere una relación estrecha entre el constructo de la impulsividad y el control inhibitorio, así, a mayor nivel de impulsividad menor control inhibitorio, sin embargo, con los hallazgos encontrados previamente por Pilatti et al. (2017), los déficits en el control inhibitorio no necesariamente indican una relación con impulsividad, debido a su carácter multidimensional que puede expresarse de forma variable de acuerdo la conducta adictiva observada.

En nuestro estudio, el IFS ha demostrado ser una buena herramienta clínica con propiedades psicométricas que muestran buena consistencia interna y validez discriminante para determinar el funcionamiento ejecutivo en adicciones; previamente, Torralva y colaboradores habían confirmado las cualidades psicométricas de la prueba en la demencia tipo alzhéimer y demencia frontotemporal (Torralva, Roca, Gleichgerrcht, Bekinschtein et al., 2009; Torralva, Roca, Gleichgerrcht, López et al., 2009), y Báez e Ibáñez reportaron las ventajas del instrumento para identificar la disfunción ejecutiva en el trastorno afectivo bipolar (Báez et al., 2014, 2017). Si bien, evaluar el funcionamiento ejecutivo en su totalidad es complejo y el uso de una sola batería neurocognitiva no permitiría sensibilizar con exactitud todos los dominios ejecutivos, el uso del IFS resulta ser una excelente herramienta clínica para el diagnóstico del funcionamiento ejecutivo, privilegiando, además, los tiempos de aplicación y la sencillez del protocolo.

Además, con respecto a la validez de clasificación, se observó la utilidad diagnóstica del test, mediante el análisis de la curva de ROC (receiveng operating characteristic), el IFS mostró una sensibilidad del $80 \%$, especificidad del $91 \%$ y un índice de discriminación de 0.93 (figura 1), que confirma la capacidad discriminativa del test para diferenciar el funcionamiento ejecutivo en grupo de policonsumidores y el grupo control, siendo el punto de corte 23. Estos resultados coinciden con las investigaciones en casos clínicos; en este sentido, el estudio de pacientes con demencia realizado por Manes y su grupo obtuvo un corte de 25 puntos, con una sensibilidad del $96.2 \%$ y especificidad del $91.5 \%$; y un área bajo la curva de 0.98 (Torralva, Roca, Gleichgerrcht, López et al., 2009). De manera similar el estudio desarrollado por Bruno y colaboradores, el uso del IFS en pacientes con esclerosis múltiple obtuvo un punto de corte de 25.5, con una sensibilidad del $73.53 \%$ y especificidad del $78.13 \%$; con una área bajo la curva de 0.85 (Bruno et al., 2015).

En coherencia con los anteriores reportes, los resultados obtenidos por el estudio de Ibáñez y su equipo evaluó la disfunción ejecutiva a través del IFS en pacientes con trastorno afectivo bipolar y trastorno por déficit de atención con hiperactividad (TDAH), y sus resultados arrojaron un punto de corte (Báez et al., 2014). El punto de corte en pacientes con trastorno bipolar fue de 27.5 puntos, con una sensibilidad del $87.5 \%$ y especificidad del 68\%; el área bajo la curva fue de 0.82 . Para el grupo TDAH y el control, el punto de corte fue de 27.5 puntos, con una sensibilidad del $80 \%$ y especificidad del $68 \%$; el área bajo la curva fue de 0.79 (Báez et al., 2014). Estudios recientes para valorar el funcionamiento ejecutivo a través del IFS en la enfermedad de Alzheimer y demencia frontotemporal variante conductual, obtuvieron un punto de corte de 23.5, sensibilidad del $97.1 \%$ y especificidad del $97.9 \%$ para la detección de alteraciones ejecutivas, con un área bajo la curva de 0.99 (Custodio et al., 2016).

Los anteriores estudios en suma con los resultados obtenidos con nuestra investigación confirman la alta capacidad del IFS para identificar el déficit en el desempeño cognitivo de personas con algún tipo de trastorno. Sin embargo, se observan dos tendencias en los puntos de corte: los estudios de pacientes con demencia, esclerosis múltiple, trastorno bipolar y TDAH cuyos puntos de corte oscilan 
entre 25-27 puntos; y el estudio de pacientes con enfermedad de Alzheimer y demencia frontotemporal variante conductual, cuyo punto de corte fue de 23.5, este punto de corte coincide con el identificado en esta investigación.

Finalmente, la batería neuropsicológica IFS constituye una excelente y versátil herramienta de tamizaje clínico que orienta el diagnóstico del funcionamiento ejecutivo y ayuda a discriminar la disfunción ejecutiva asociada con la dependencia de sustancias psicoactivas; asimismo, delinea vías alternativas de rehabilitación neurocognitiva en este tipo de poblaciones, en pro de mejorar la inserción social y el desempeño funcional de los pacientes.

\section{Referencias}

Acosta-Barreto, M. R., Juárez-Acosta, F., \& Cuartas-Arias, M. (2018). Funciones ejecutivas y antecedentes familiares de alcoholismo en adolescentes. Pensamiento Psicológico, 16(1), 57-68.

Báez, S., Ibáñez, A., Gleichgerrcht, E., Pérez, A., Roca, M., Manes, F., \& Torralva, T. (2014). The utility of IFS (INECO frontal screening) for the detection of executive dysfunction in adults with bipolar disorder and ADHD. Psychiatry Research, 216(2), 269276. http://dx.doi.org/10.1016/j.psychres.2014.01.020

Báez, S., Pinasco, C., Roca, M., Ferrari, J., Couto, B., García-Cordero, I., \& Torralva, T. (2017). Brain structural correlates of executive and social cognition profiles in behavioral variant frontotemporal dementia and elderly bipolar disorder. Neuropsychologia, 126,159-169. https://dx.doi.org/10.1016/j.neuropsychologia.2017.02.012

Bruno, D., Torralva, T., Marenco, V., Ardilla, J. T., Báez, S., Gleichgerrcht, E., ..., \& Roca, M. (2015). Utility of the INECO frontal screening (IFS) in the detection of executive dysfunction in patients with relapsing-remitting multiple sclerosis (RRMS). Neurological Sciences, 36(11), 2035-2041. http://dx.doi. org/10.1007/s10072-015-2299-6

Burgess, P. W., \& Shallice, T. (1997). The Hayling and Brixton tests. Thurston, Suffolk: Thames Valley Test Company.

Buriel, Y., Gramunt, N., Bohm, P., Rodes, E., \& Peña-Casanova, J. (2004). Verbal fluency: Preliminary normative data in a Spanish sample of young adults (20-49 years of age). Neurología, 19(4), 153-159.

Cunha, P. J., Nicastri, S., de Andrade, A. G., \& Bolla, K. I. (2010). The frontal assessment battery (FAB) reveals neurocognitive dysfunction in substance-dependent individuals in distinct executive domains: Abstract reasoning, motor programming, and cognitive flexibility. Addictive Behaviors, 35(10), 875-881. http://dx.doi.org/10.1016/j.addbeh.2010.05.005

Custodio, N., Herrera-Pérez, E., Lira, D., Roca, M., Manes, F., Báez, S., \& Torralva, T. (2016). Evaluation of the INECO frontal screening and the frontal assessment battery in Peruvian patients with Alzheimer's disease and behavioral variant frontotemporal dementia. eNeurologicalSci, 5, 25-29.

De Melo-Rodrigues, L. C., Conti, C. L., \& Nakamura-Palacios, E. M. (2011). Clozapine and SCH 23390 prevent the spatial working memory disruption induced by $\triangle 9$-THC administration into the medial prefrontal cortex. Brain Research, 1382, 230-237. http://dx.doi.org/10.1016/j.brainres.2011.01.069

Dubois, B., Slachevsky, A., Litvan, I., \& Pillon, B. F. A. B. (2000). The FAB: A frontal assessment battery at bedside. Neurology, 55(11), 1621-1626.

Fernández, G., García, O., Secades, R., Álvarez, J., \& Sánchez, E. (2008). Rendimiento neuropsicológico de pacientes en tratamiento por adicción a la cocaína. Salud y Drogas, 11-28.

Fernández-Montalvo, J., López-Goni, J. J., Azanza, P., Arteaga, A., \& Cacho, R. (2017). Gender differences in treatment progress of drug-addicted patients. Women Health, 57(3), 358-376. http://dx.doi.org/10.1080/03630242.2016.1160967
Fisher, W. P. Jr. (2011). Stochastic and historical resonances of the unit in physics and psychometrics. Measurement: Interdisciplinary Research \& Perspectives, 9(1), 46-50. http://dx.doi.org/1 $0.1080 / 15366367.2011 .558789$

Hodges, J. R. (1994). Cognitive assessment for clinicians. Oxford: Oxford University Press.

Hser, Y. I., Huang, D., Teruya, C., \& Anglin, M. D. (2003). Gender comparisons of drug abuse treatment outcomes and predictors. Drug \& Alcohol Dependence, 72(3), 255-264.

Ihnen, J., Antivilo, A., Muñoz-Neira, C., \& Slachevsky, A. (2013). Chilean version of the INECO frontal screening (IFS-Ch): Psychometric properties and diagnostic accuracy. Dementia \& Neuropsychologia, 7(1), 40-47.

Karr, J. E., Areshenkoff, C. N., Rast, P., Hofer, S. M., Iverson, G. L., \& García-Barrera, M. A. (2018). The unity and diversity of executive functions: A systematic review and re-analysis of latent variable studies. Psychological Bulletin, 144(11), 1147. http:// dx.doi.org/10.1037/bul0000160

López-Larrosa, S., \& Rodríguez-Arias, J. L. (2010). Factores de riesgo y de protección en el consumo de drogas en adolescentes y diferencias según edad y sexo. Psicothema, 22(4).

López-Larrosa, S., \& Rodríguez-Arias, J. L. (2012). Factores de riesgo y de protección en el consumo de drogas y la conducta antisocial en adolescentes y jóvenes españoles. International Journal of Psychological Research, 5(1), 25-33.

Miyake, A., Friedman, N. P., Emerson, M. J., Witzki, A. H., Howerter, A., \& Wager, T. D. (2000). The unity and diversity of executive functions and their contributions to complex "Frontal Lobe" tasks: A latent variable analysis. Cognitive Psychology, 41(1), 49-100.

Muñiz, J. (2000). Teoría clásica de los tests. Madrid: Pirámide.

Muñiz, J., Elosua, P., \& Hambleton, R. K. (2013). Directrices para la traducción y adaptación de los tests. Psicothema, 25(2).

Noël, X., Brevers, D., \& Bechara, A. (2013). A neurocognitive approach to understanding the neurobiology of addiction. $\mathrm{Cu}$ rrent Opinion in Neurobiology, 23(4), 632-638. http://dx.doi. org/10.1016/j.conb.2013.01.018

Organización de Naciones Unidas. Oficina de las Naciones Unidas contra la Droga y el Delito (UNODC). (2017). Informe mundial sobre las drogas. Subdivisión de Investigación y Análisis de Tendencias. Viena: UNODC. Informe mundial sobre las drogas 2017: resumen, conclusiones y consecuencias en materia de políticas.

Perry, C. J., \& Lawrence, A. J. (2017). Addiction, cognitive decline and therapy: Seeking ways to escape a vicious cycle. Genes, Brain and Behavior, 16(1), 205-218. http://dx.doi.org/10.1111/ gbb.12325

Pilatti, A., Montejano, G. R., Cesar, A., Bertone, M. F. H., Cumin, G., \& Pautassi, R. M. (2017). Efecto del estrés social agudo sobre impulsividad, toma de riesgos y sesgos atencionales en jóvenes con y sin historia familiar de abuso de alcohol. Suma Psicológica, 24(2), 115-128. http://dx.doi.org/10.1016/j.sumpsi.2016.09.003

Prieto, G., Delgado, A. R., Perea, M. V., \& Ladera, V. (2010). Scoring neuropsychological tests using the Rasch model: An illustrative example with the Rey-Osterrieth complex figure. The Clinical Neuropsychologist, 24(1), 45-56. http://dx.doi. org/10.1080/13854040903074645

Secades-Villa, R., \& Fernández-Hermida, R. (2003). Guía de los tratamientos psicológicos eficaces para la drogadicción: alcohol, cocaína y heroína. En Pérez, M., Fernández-Hermida, J. R., Fernández, C., \& Amigo, I. Guía de tratamientos psicológicos eficaces (pp. 107-139). Madrid: Pirámide.

Smith, D. G., Jones, P. S., Bullmore, E. T., Robbins, T. W., \& Ersche, K. D. (2013). Cognitive control dysfunction and abnormal frontal cortex activation in stimulant drug users and their biological siblings. Translational Psychiatry, 3(5), e257. http://dx.doi. org/10.1038/tp.2013.32 
Torralva, T., Roca, M., Gleichgerrcht, E., Bekinschtein, T., \& Manes, F. (2009). A neuropsychological battery to detect specific executive and social cognitive impairments in early frontotemporal dementia. Brain, 132(5), 1299-1309. http://dx.doi. org/10.1093/brain/awp041

Torralva, T., Roca, M., Gleichgerrcht, E., López, P., \& Manes, F. (2009). INECO frontal screening (IFS): A brief, sensitive, and specific tool to assess executive functions in dementia. Journal of the International Neuropsychological Society, 15(5), 777786. http://dx.doi.org/10.1017/S1355617709990415

Fisher, W. P. Jr. (2011). AERA-APA-NCME standards for educational and psychological testing. Rasch Measurement Transactions, 24(4), 1310.
Wechsler, D. (1987). Wechsler memory scale-revised. New York: Psychological Corporation.

Wesley, M. J., Lile, J. A., Fillmore, M. T., \& Porrino, L. J. (2017). Neurophysiological capacity in a working memory task differentiates dependent from nondependent heavy drinkers and controls. Drug \& Alcohol Dependence, 175, 24-35. http://dx. doi.org/10.1016/j.drugalcdep.2017.01.029

Yücel, M., Lubman, D. I., Solowij, N., \& Brewer, W. J. (2007). Understanding drug addiction: A neuropsychological perspective. Australian \& New Zealand Journal of Psychiatry, 41(12), 957968. http://dx.doi.org/10.1080/00048670701689444 